\title{
OBSERVACIONES PRELIMINARES SOBRE COMPORTAMIENTO PRENATAL Y POSTNATAL DE Rattus rattus var. norvegicus (Linneo 1758) EN EL LABORATORIO
}

Martha Williams de Castro ${ }^{1}$, Alexandra Castillo ${ }^{2}$ y Constanza Rosas ${ }^{3}$

\section{Resumen}

Con el objeto de lograr entrenamiento en la observación de roedores silvestres traídos al laboratorio, se llevó a cabo un estudio del comportamiento durante la preñez y lactancia de Rattus rattus var. norvegicus (Linneo 1758). Se observó que la agresividad de la madre aumentó conforme iba acercándose el parto, el cual se produjo al décimo séptimo día de gestación. Desde que las crías nacieron, la madre se mostró muy celosa y protectora; y nunca rechazó a sus crías a pesar de que estas eran manipuladas diariamente, para evaluar su peso y sus medidas de longitud cabeza - cuerpo. No se presentó canibalismo. El éxito reproductivo fue del $100 \%$ pues todas las crías llegaron a la adultez. Para el peso se encontró que las diferencias sexuales se hacen significativas a partir del día $29(\mathrm{p}=0.006)$ mientras que para la longitud cabeza- cuerpo ocurre a partir del día $32(\mathrm{p}=0.012)$. El destete se produjo el día 30 .

Palabras clave: Comportamiento maternal, gestación, parto, lactancia, destete, éxito reproductivo, bioterio.

\begin{abstract}
The present research work was conducted with the objective of acquiring training in the observation of wild rats brought to laboratory conditions. It consisted in observing pre and post natal behavior in Rattus rattus var. norvegicus (Linneo 1758). The fact that the mother's aggressiveness increased with the arrival of parturition, event that occurred on the 17th day of gestation was observed. Once the pups were born, the mother showed herself protective and careful. She never rejected her pups even when they were manipulated daily to assess their weight and head - body size development. Cannibalism was not observed. The reproductive success was a $100 \%$ considering the fact that all pups reached adulthood. In relation to weight and head -body size, it was found that sexual differences became significant around day $29^{\text {th }}(p=0.006)$ and day $32^{\text {nd }}(p=0.012)$, respectively. Weaning occurred on day $30^{\text {th }}$.
\end{abstract}

Key words: Maternal behavior, gestation, parturition, lactation, weaning, reproductive success, rearing center.

\section{Introducción}

La rata blanca de laboratorio Rattus rattus var. norvegicus, goza de general aceptación para el estudio del comportamiento. Entre las ventajas de trabajar con esta especie destacan: su tamaño pequeño, su limpieza, facilidad de manejo y de alojamiento, y el bajo costo de su mantenimiento (Munn, 1950). Por su ciclo de vida corto, es ideal para realizar estudios de comportamiento materno. Esta especie alcanza la madurez sexual entre los 60 y 90 días, y posee un periodo de gestación que dura de 22 a 24 días, al final del cual nacen de 8 a 12 individuos. Puede tener de 8 a 12 nidadas por año y tiene una vida media de dos años (Vega, 2000).

En el presente trabajo, realizado en el Laboratorio de Fisiología Animal de la UNALM, se hacen observaciones del comportamiento de una rata madre durante los periodos de gestación, lactancia y destete; y del comportamiento de sus once crías. Además, se comparan los datos de la evolución del peso y el crecimiento cabeza - cuerpo de las mismas. Con esta investigación se busca establecer patrones de conducta tanto de la madre como de las crías y establecer cómo se va desarrollando el grado de diferencia en peso y tamaño entre hembras y machos.

El entrenamiento con Rattus rattus var. norvegicus es de gran importancia, ya que brinda la experiencia para posteriormente realizar estudios con roedores silvestres de los que su biología se sabe muy poco. Para citar unos ejemplos, el entrenamiento posibilita realizar estudios como el de Künkele (2000) que muestra los efectos del tamaño de camada en la energética reproductiva de Cavia porcellus, o el estudio de Veloso \& Bozinovic (2000) que examina los efectos de la calidad de alimento en la energética reproductiva de Octodon degus; o finalmente el

\footnotetext{
${ }^{1}$ Laboratorio de Fisiología Animal y Biorremediación. Departamento de Biología, Universidad Nacional Agraria La Molina. Apartado 456. Lima 100. Correo electrónico: Mwilliams@lamolina.edu.pe

2 Departamento de Biología, Universidad Nacional Agraria La Molina. Apartado 456. Lima 100. Correo electrónico: 19960862@lamolina.edu.pe

${ }^{3}$ Departamento de Biología, Universidad Nacional Agraria La Molina. Apartado 456. Lima 100. Correo electrónico: 19970113@lamolina.edu.pe
} 
estudio de Sumbera et al. (2003) en el que por primera vez se reporta aspectos de la biología de Heliophobius argenteocinereus.

\section{Revisión de Literatura}

A diferencia de otras especies que requieren experiencias previas para mostrar un cuidado materno adecuado, las ratas hembras de Rattus rattus var. norvegicus bajo condiciones de laboratorio, y sin ninguna experiencia previa con crías, usualmente cuidan de forma adecuada a su primera camada. La madre inexperta construye un nido, alumbra, reúne, limpia, y atiende a sus crías bajo la influencia de los niveles cambiantes de las hormonas (Fleming et al., 1999).

Las hormonas juegan un rol muy importante en el desencadenamiento del comportamiento materno. En los últimos días de gestación, los niveles de oxitocina cambian dramáticamente en áreas del cerebro relacionadas con la conducta materna y retornan a la normalidad días después del parto. El contacto físico entre madre y crías, particularmente el que se da en la lactancia, es el que sigue desencadenando el comportamiento materno (Pedersen, 1979, 1992, citado por Zubritsky, 1997). Otras hormonas relacionadas con el comportamiento materno son: la progesterona que disminuye durante los últimos días de gestación, y el estrógeno y la prolactina que incrementan justo antes del parto. Estas hormonas pueden preparar lugares que regulen el aprendizaje y la memoria, y estimular el área préoptica media para responder con cuidado maternal al nacimiento de sus crías (Loy \& Gerlach, 1988; Numan, 1991,citados por Kinsley et al., 1999). Además, las hormonas prolactina y la oxitocina son necesarias para la producción de leche.

La influencia hormonal y la exposición a las crías, hacen que la madre involucre respuestas motoras ante ellas, como el levantarlas, movilizarlas hacia sitios seguros y colocarse sobre ellas (Fleming et al., 1999); además de evitar el hacerles daño (Peters et al., 1991). La sensibilidad hacia las crías aún se mantiene alta, semanas después del destete.

El comportamiento materno también incluye la desaparición de conductas infanticidas que han sido observadas en ratas nulíparas y otras que ya han tenido crías pero que no se encuentran gestando. La desaparición del infanticidio se da tiempo antes del parto, y se presume que también está influenciado por factores fisiológicos (Peters \& Cristal, 1983; citados por Peters et al., 1991).

Después de un período promedio de gestación de 21 días, poco antes del parto, la madre comienza la construcción del nido. Utiliza de entre todos los materiales que se encuentran a su disposición, los que han estado relacionados con ella en alguna época de su vida (Riess, citado por Barnett, 1963). Barnett (1963) afirma que la construcción del nido es una actividad homeostática ya que contribuye a mantener constante la temperatura corporal de las crías.

Antes del parto, el dolor abdominal en la madre producido por las contracciones uterinas intensas la obliga a ponerse en posición de cúbito abdominal y a estirar las patas del tren posterior (Vega, 2000). El número de crías nacidas por parto fluctúa entre 8 y 12 crías (Shively, 1993; Vega, 2000). La madre ayuda a las crías a salir jalándolas con los dientes. Luego ingiere la placenta, el cordón umbilical y se deshace de las membranas fetales de las crías, con la finalidad de estimularlas y eliminar el contenido líquido de las vías respiratorias de los recién nacidos.

Después de nacer, las crías desarrollan una atracción química hacia el vientre materno y se adhieren a los pezones como consecuencia de la experiencia intrauterina con el líquido amniótico. La atracción a menudo es seguida por el desarrollo de preferencias en los pezones. El reconocimiento de la madre se da por el olor que ella emana (Pederson \& Blass, 1981; citados por Fleming et al., 1999). La estimulación causada por la lactancia provoca que la leche sea expulsada directamente dentro de la boca de las crías.

Las crías recién nacidas tienen un peso de 5-6 gramos, nacen sin pelo, ciegas y sordas (Vega, 2000). Emiten dos tipos de ultrasonidos. El primero en respuesta al frío, desencadena la actitud de búsqueda por parte de la madre. El segundo en respuesta a un trato brusco de la madre, causa que ésta deje de provocar el estímulo que molesta a la cría.

Alrededor del día 7 después del nacimiento el pelo comienza a hacerse más visible. Entre los días 14 y 17, los ojos y oídos comienzan a abrirse (Shively, 1993) y las crías se hacen más móviles. La hiperactividad se debe a que las áreas excitatorias del cerebro asociadas con la actividad ya han madurado pero las áreas inhibitorias de la actividad no lo han hecho aún. La maduración recién se da a inicios de la tercera semana (día 21). El destete también se produce alrededor del día 21 ya que la madre experimenta dolor en los pezones y por la presencia de pelo en las crías.

\section{Materiales y métodos}

En el mes de mayo del 2001 se inició el experimento con una rata madre preñada (segunda camada), criada en un bioterio. Antes del parto la madre fue puesta en una jaula $(29$ × 29 × $29 \mathrm{~cm})$ y todos los días se le administraba agua ad libitum además de $40 \mathrm{~g}$ de alimento balanceado Tipo I (Tabla 1) antes del nacimiento de las crías y luego del Tipo II (Tabla 1) después del nacimiento. La madre fue pesada tres veces antes del parto para su control. El noveno día después del parto se trasladaron madre y crías a otro ambiente (54x 29.5x $29 \mathrm{~cm})$ para su seguridad y comodidad, ya que se buscaba proveer a los animales de un ambiente que los enriqueciera (Chmiel \& 
Noonan, 1996). Por último, en la tercera semana después del nacimiento, se colocaron en el ambiente una serie de trampolines y juegos para la estimulación mental y el desarrollo de conductas específicas de la especie.

Tabla 1. Composición de las dietas utilizadas en la experimentación (Tipo I y II) y de dietas que muestran las composiciones nutricionales recomendadas.

\begin{tabular}{|c|c|c|c|}
\hline Componentes & $\begin{array}{l}\text { Dieta } \\
\text { Tipo I a }\end{array}$ & $\begin{array}{l}\text { Dieta } \\
\text { Tipo II b }\end{array}$ & $\begin{array}{l}\text { Composiciones } \\
\text { nutricionales } \\
\text { recomendadas }\end{array}$ \\
\hline \multicolumn{4}{|l|}{ Dieta Básica } \\
\hline Materia seca (\%) & & 88.433 & $96.900 \mathrm{c}$ \\
\hline Proteína (\%) & 21.000 & 17.216 & $14.700 \mathrm{c}$ \\
\hline Fibra (\%) & 5.000 & 3.849 & $7.400 \mathrm{c}$ \\
\hline Grasa (\%) & 4.000 & & $5.000 \mathrm{c}$ \\
\hline Ceniza (\%) & 8.000 & & $3.700 \mathrm{c}$ \\
\hline Extracto etéreo (\%) & & 4.363 & $6.800 \mathrm{c}$ \\
\hline EM. Aves Mcal/kg & & 2.880 & \\
\hline \multicolumn{4}{|l|}{ Aminoácidos } \\
\hline Lis $(\%)$ & & 0.920 & $0.920 \mathrm{~d}$ \\
\hline Met (\%) & & 0.622 & \\
\hline Met -Cis (\%) & & 0.980 & $0.980 \mathrm{~d}$ \\
\hline $\operatorname{Arg}(\%)$ & & 1.075 & $0.430 \mathrm{~d}$ \\
\hline Treo $(\%)$ & & 0.620 & $0.620 \mathrm{~d}$ \\
\hline Trip (\%) & & 0.227 & $0.200 \mathrm{~d}$ \\
\hline Gli- Ser (\%) & & 1.681 & \\
\hline His (\%) & & 0.457 & $0.280 \mathrm{~d}$ \\
\hline Ile $(\%)$ & & 0.742 & $0.620 \mathrm{~d}$ \\
\hline Leu $(\%)$ & & 1.411 & $1.070 \mathrm{~d}$ \\
\hline Fen $(\%)$ & & 0.822 & \\
\hline Fen-Tir (\%) & & 1.390 & $1.020 \mathrm{~d}$ \\
\hline $\operatorname{Val}(\%)$ & & 0.852 & $0.740 \mathrm{~d}$ \\
\hline \multicolumn{4}{|l|}{ Minerales } \\
\hline Fósforo (\%) & & 0.370 & $0.300 \mathrm{~d}$ \\
\hline $\mathrm{Ca}(\%)$ & & 0.630 & $0.500 \mathrm{~d}$ \\
\hline $\mathrm{Na}(\%)$ & & 0.050 & $0.500 \mathrm{~d}$ \\
\hline
\end{tabular}

(a) Composición del alimento balanceado Iniciarina Reforzada suministrado a la madre durante el periodo de gestación.

(b) Composición del alimento para ratas suministrado a la madre durante el periodo de lactancia.

(c) Valores hallados en una ración que cubría los requerimientos nutricionales de las ratas. Fuente: Gutiérrez, 2001.

(d) Requerimientos nutricionales estimados para el crecimiento de las ratas. Fuente: National Research Council, 1995.

Un día después del nacimiento de las crías (5 hembras y 6 machos) se inició la evaluación diaria del peso y la evaluación interdiaria de la longitud cabeza cuerpo. Para la identificación de las crías se ideó un sistema de marcación en el cual las uñas de las hembras fueron pintadas con rojo y la de los machos con azul.

Se anotaron todos los patrones de comportamiento de la madre y de las crías, así como la evolución de las características físicas, hasta el día en que madre e hijos fueron completamente aislados. Para efectos del análisis estadístico de los datos obtenidos de las crías, se usó la prueba $\mathrm{T}$ tanto para evaluar peso como longitud cabeza-cuerpo.

\section{Resultados}

\section{Comportamiento de madre y crías}

Desde un comienzo, la rata madre se mostró agresiva y fue difícil su manipulación. Se esperaba que las crías nacieran al día 21 de gestación mas el nacimiento se produjo al día 17 . Horas antes del inicio del parto, la madre empezó con la construcción del nido. Para la construcción de éste, la madre mostró preferencia por el papel periódico a pesar de haber tenido al alcance otros materiales, como pedazos de tela y lana. Cuando se trató de incorporar éstos últimos al nido, la madre reaccionaba rápidamente, y los alejaba. Usó todo el papel periódico de la jaula formando una especie de túnel al que le clausuró todas las entradas.

La madre parió 11 crías ( 5 hembras y 6 machos), a las que cuidó y protegió celosamente sin hacer distinción por alguna en particular. La madre trataba de mantener a las crías en el nido todo el tiempo. Cada vez que una cría intentaba acercarse a las experimentadoras la madre la tomaba cuidadosamente con sus dientes y la colocaba dentro del nido rápidamente. Este comportamiento se observó aún después de que las crías se mostraran independientes; es decir cuando los ojos y oídos ya estaban abiertos y podían desplazarse con confianza. En la exploración del ambiente, las hembras se mostraron más curiosas que los machos y en el inicio de los primeros signos de juego, las hembras se mostraron más activas que los machos.

En cuanto al alimento y agua consumidos por la madre durante la gestación y lactancia, se observó un mayor consumo en este último periodo. Debido a ello se le administró el alimento Tipo II (Tabla 1), dieta alta en proteínas formulada especialmente para ratas.

\section{Desarrollo de las características físicas de las crías}

En la Tabla 1 se compara la concentración de componentes de las dietas Tipo I y II con las concentraciones de componentes de otras dietas que se sabe satisfacen los requerimientos nutricionales de las ratas (National Research Council, 1995; Gutiérrez, 2001). Se puede observar que la concentración de los componentes de las dietas utilizadas en este experimento, excede a la concentración de las dietas recomendadas, porque en la elaboración de las dietas se busca un margen de seguridad.

En la Tabla 2 se muestra un resumen de la aparición de ciertas características físicas y de comportamiento de las crías. Las crías nacen sin pelo, dientes, con los ojos y oídos cerrados, dependiendo completamente del cuidado de la madre. La aparición del pelo en promedio comienza a partir del séptimo día, presentándose como una especie de pelusa la cual 
alrededor del día 11 ya es un pelo grueso y blanco brilloso. Para esta última fecha los dientes ya están formados. Los ojos y oídos se abren alrededor del día 15 , siendo éste el comienzo de la exploración de su ambiente, el cual ya reconocen muy bien por el olfato. Empiezan a aproximarse a la comida y al agua como parte de un juego y a partir del día 17 después del nacimiento ya se alimentan solas. Las hembras se muestran más curiosas y avezadas que los machos para explorar lo que se encuentra fuera de su nido y especialmente los juegos y trampolines dispuestos para propiciar la estimulación mental

Tabla 2. Características físicas y comportamiento de las crías de Rattus rattus var norvegicus, observaciones efectuadas en el Laboratorio del Fisiología Animal UNALM; del 23 de mayo (día 7) al 12 de junio del 2001 (día 30).

\begin{tabular}{|c|c|c|c|c|c|c|c|}
\hline & 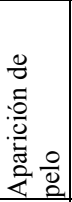 & 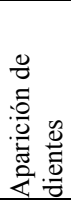 & 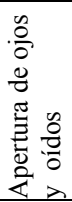 & 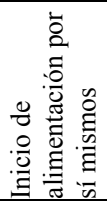 & 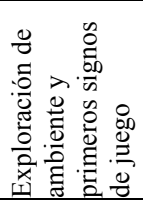 & 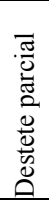 & 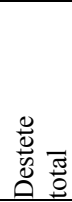 \\
\hline $\begin{array}{l}\text { Día } \\
7\end{array}$ & $X$ & & & & & & \\
\hline \begin{tabular}{|l} 
Día \\
11
\end{tabular} & & $X$ & & & & & \\
\hline $\begin{array}{l}\text { Día } \\
15\end{array}$ & & & $X$ & & & & \\
\hline \begin{tabular}{|l|} 
Día \\
17 \\
\end{tabular} & & & & $X$ & $X$ & & \\
\hline $\begin{array}{l}\text { Día } \\
22\end{array}$ & & & & & & $X$ & \\
\hline $\begin{array}{l}\text { Día } \\
30\end{array}$ & & & & & & & $X$ \\
\hline
\end{tabular}

El día 22 después del nacimiento se separó la madre de 9 de sus crías. Con este destete parcial se evita problemas de mastitis con la madre. El día 30 se produjo el destete total y hasta esta fecha se observaron cuidados maternales. Como producto de la separación la madre se mostró inapetente y redujo su actividad.

A pesar de que el canibalismo es un fenómeno que ocurre muy frecuentemente en roedores criados en bioterios, en este caso no se presentó.

\section{Peso:}

Desde el nacimiento se observó que los machos tienden a tener mayor peso que las hembras. La prueba $\mathrm{T}$ utilizada muestra que aproximadamente a fines de la cuarta semana (día 29) se comienzan a expresar significativamente la diferencia de peso entre hembras y machos $(p=0.006)$. Para el análisis de datos se trabajó con los datos obtenidos a partir del día 5 después del nacimiento pues fue para entonces cuando se pudo realizar el sexado correspondiente.

El crecimiento tanto de las hembras como de los machos puede modelarse con una función sigmoidea.
Esto concuerda con lo observado por Zullinger et al. (1984): "...el aumento de peso en los mamíferos no es lineal ... las curvas de crecimiento de muchas de las especies son de forma sigmoidal". Los resultados de peso, ajustados a la curva sigmoidal de Gompertz arrojaron los siguientes valores: $\mathrm{y}=449.292 * \mathrm{e}^{\wedge}\left(-\mathrm{e}^{\wedge}(-\right.$ $0.037(\mathrm{x}-45.65))$ ) con un $\mathrm{R}^{2}$ de 0.996279 para los machos y la siguiente ecuación $\mathrm{y}=269.292 * \mathrm{e}^{\wedge}\left(-\mathrm{e}^{\wedge}(-\right.$ $0.045(\mathrm{x}-36))$ ) con un $\mathrm{R}^{2}$ de 0.9941 para las hembras, en las cuales e es la base del logaritmo neperiano, la variable dependiente es el peso en gramos y la independiente es el tiempo en días. La Figura 1 muestra las líneas de tendencia, observándose que las diferencias entre los sexos van acentuando conforme va aumentando el peso.

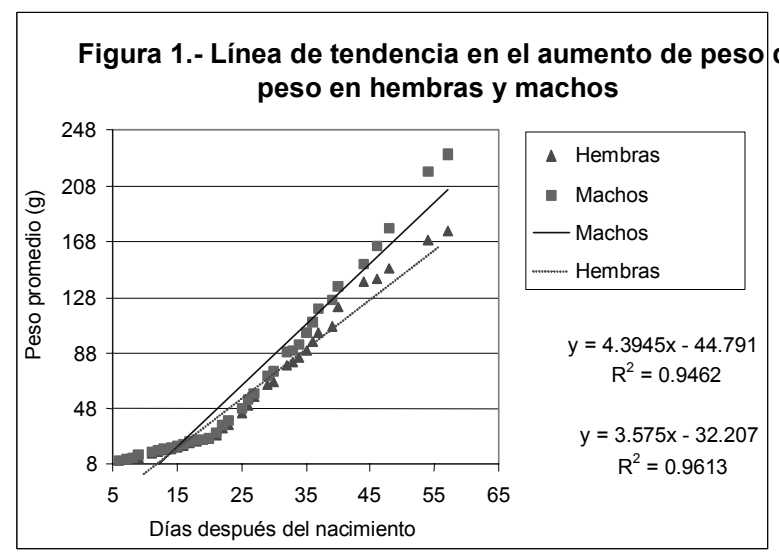

\section{Longitud cabeza - cuerpo:}

Las dimensiones de longitud cabeza-cuerpo también se ajustan a la ecuación de Gompertz arrojando los siguientes valores: $\mathrm{y}=42.12 * \mathrm{e}^{\wedge}\left(-\mathrm{e}^{\wedge}(-\right.$ $0.0219(\mathrm{x}-37.37)))$ con un $\mathrm{R}^{2}$ de 0.99123 para los machos y la siguiente ecuación y $=30.337 * \mathrm{e}^{\wedge}\left(-\mathrm{e}^{\wedge}(-\right.$ $0.027(\mathrm{x}-25.38))$ ) con un $\mathrm{R}^{2}$ de 0.986398 para las hembras, en las cuales e es la base del logaritmo neperiano, la variable dependiente es la longitud en centímetros y la independiente es el tiempo en días. $\mathrm{Al}$ nacer, las crías no muestran diferencias en el tamaño, pero a partir de la segunda semana, los machos muestran un mayor tamaño que las hembras (Figura 2). La línea de tendencia muestra con un $R^{2}$ de 0.9855 para los machos y 0.9826 para las hembras, que las diferencias en tamaño se van acentuando conforme van adquiriendo mayor tamaño. La prueba $T$ realizada mostró diferencias significativas en las diferencias de longitudes entre hembras y machos alrededor del día $32(p=0.012)$. Esto concuerda con lo ocurrido con el peso, que también se hace significativo alrededor de esta fecha.

\section{Discusión}

Las ratas adquiridas en centros de crianza o bioterios muestran un comportamiento agresivo, ya que se desarrollan en espacios muy pequeños y el 
contacto con los humanos es mínimo. Esta agresividad es aún mayor si la rata se encuentra gestando o lactando. Debido a todo ello es comprensible que la rata del experimento se mostrara en extremo agresiva y de difícil manipulación.

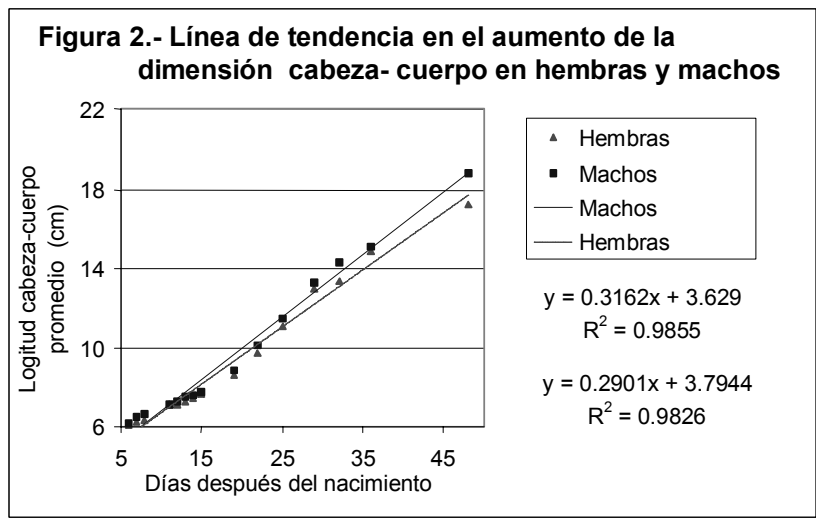

La preferencia de la madre por papel periódico en la construcción del nido podría deberse a que éste es uno de los elementos con los que más se ha relacionado durante toda su vida ya que nació, creció y tuvo su primera camada en un ambiente compuesto únicamente por papel periódico. Riess (citado por Barnett, 1963) sugirió que la construcción del nido depende de un tipo de experiencia específico en un estadío de vida temprano. El mencionado autor crió ratas en un ambiente carente de elementos. Cuando estas ratas tuvieron que construir un nido, no lo hicieron a pesar de tener material disponible. En este estudio, la preferencia por el papel periódico puede interpretarse como la preferencia por un elemento con el que la madre se siente relacionada y por tanto le da suficiente seguridad para depositar a sus crías pues ella ya conoce las potencialidades del elemento (imprinting).

Según Shively (1993) el parto de las ratas se produce entre el día 21 y día 23 de gestación pero en este caso se produjo en el día 17. Este adelanto pudo deberse a dos factores: (i) mayor actividad física producto de un área más amplia de desenvolvimiento, y (ii) estrés producido por el cambio de ambiente y el traslado continuo hacia diferentes áreas de trabajo.

El comportamiento dedicado de la madre hasta días después del destete es coincidente con lo encontrado en la literatura (Steuer et al., 1987; Peters et al., 1991; Fleming et al., 1999), en la que se explica que este comportamiento está regulado por los cambios de niveles hormonales, principalmente por los cambios de cuatro hormonas: la oxitocina, el estrógeno, la progesterona y la prolactina.

Muchos estudios de la energética reproductiva en mamíferos han confirmado que la reproducción es el periodo de vida que demanda mayor cantidad de energía (Bronson, 1989; citado por Veloso \& Bozinovic, 2000). Especialmente durante la lactancia el gasto de energía es muy alto (Millar, 1979; Oftedal, 1984; Thompson \& Nicoll, 1986; Kenagy, 1987; 1989: McClure, 1987; Hayes et al., 1992; Thompson, 1992; citados por Veloso \& Bozinovic, 2000). Una malnutrición durante la gestación y lactancia propicia que las crías nazcan con bajo peso, que estas sean más propensas a las enfermedades y a la muerte y que las madres produzcan menor cantidad y calidad de leche (Stylianopoulos, 1999). Es por ello que se buscó alimentos enriquecidos para alimentar a la madre y a sus crías. Antes del parto se alimentó a la madre con un alimento formulado para pollos (Tipo I) y en una etapa postnatal se alimento a todas las ratas con el alimento Tipo II. El cambio de dieta se debió a que el segundo era formulado específicamente para ratas. Ambos tipos de alimento tenían un alto contenido proteico (Tabla 1) y cubrían satisfactoriamente los requerimientos nutricionales para el crecimiento de las crías.

La calidad de la dieta en la etapa prenatal y postnatal fue factor muy importante para la obtención de $92 \%$ de preñez (si se considera que 12 es la mayor cantidad de crías obtenidas por camada; Shively, 1993; Vega, 2000) y de $100 \%$ de éxito reproductivo, ya que la totalidad de crías llegaron a la adultez.

La proteína brindada en la dieta sería una de las explicaciones a la ausencia de canibalismo. La madre obtenía suficiente proteína y no necesitaba una cantidad adicional proveniente de sus crías. Otra de las explicaciones sería la reducción en el estrés producido por el ambiente, ya que el área del nuevo ambiente proporcionado triplicaba las dimensiones del área donde la rata madre había vivido.

La dieta también habría influido enormemente en el desarrollo del fenotipo, particularmente en la expresión del pelo. Estudios realizados en ratas con tipos diferentes de dietas: pobres y enriquecidas en nutrientes (Stylianopoulos, 1999), mostraron que el pelo de las ratas expuestas al primer tratamiento era áspero y opaco. Mas aún, en algunos casos llegaban a perder el pelo y desarrollaban dermatitis. En cambio las ratas alimentadas con la dieta enriquecida con formulaciones muy parecidas a los alimentos tipo I y II; mostraban el pelo liso y brillante, lo cual concuerda con nuestro alimento Tipo II, ya que las ratas mostraron un pelo muy brilloso.

\section{Conclusiones}

Los elementos con los que la madre estuvo relacionada en algún momento de su vida fueron los que escogió para la construcción de su nido.

El alimento usado cubrió los requerimientos alimenticios de la madre durante la preñez y lactancia, así como también los requerimientos nutricionales de las crías durante su desarrollo. Esto se comprueba con el $100 \%$ de supervivencia de las crías.

Como en la mayoría de mamíferos, en las ratas el dimorfismo sexual está dirigido a los machos. Estos 
últimos presentan mayor peso y tamaño que las hembras. Las diferencias de dichas magnitudes entre los sexos son muy pequeñas al nacer y se hacen significativas al primer mes de vida.

Durante todo el experimento se observaron comportamientos normales tanto de la madre como de la crías, observándose que para el juego, las hembras se mostraban más curiosas y activas que los machos.

Mediante este experimento se ha logrado obtener el entrenamiento buscado en la observación del comportamiento prenatal y postnatal de roedores de laboratorio que servirá de base para la observación del comportamiento de roedores silvestres.

\section{Agradecimientos}

A Erika Paliza y a Mirtha Yrivarren por su apoyo y ayuda incondicional.

\section{Bibliografía}

Barnett S.A. 1963. The Rat a study in Behaviour. Aldine Publishing Company. Chicago - USA.

Chmiel D.J. \& Noonan M. 1996. Preference of Laboratory Rats for Potentially Enriching Stimulus Objects. Laboratory Animals. 30: 97-101.

Fleming O.S., O'Day D.H. \& Kraemer G.W. 1999. Neurobiology of mother infant interactions: experience and central nervous system plasticity across development and generations. Neuroscience and Biobehavioral Reviews. 23: 673- 685.

Gutierrez C. 2001. Comparación de Tres Métodos en la Determinación de la Digestibilidad de una Dieta Estándar en Rattus norvergicus. Journal Boliviano de Ciencias 3 (6):9-9

Kinsley C.H., Madonia L., Gifford G.W., Tureski K., Griffin G.R., Lowry C., Williams J., Collins J., MClearie H. \& Lambert K.G.1999. Motherhood improves learning and memory. Nature. 402 (11): $137-138$.

Künkele J. 2000. Effects of litter size on the energetics of reproduction in a highly precocial rodent, the Guinea Pig. Journal of Mammalogy. 81 (3): 691700.

Munn N.L. 1950. Handbook of Psychological Research on the Rat. The Riverside Press. Cambridge Massachusetts. USA.

NATIONAL RESEARCH COUNCIL. 1995. Nutrient Requirements of Laboratory Animals. Fourth
Revised Edition. National Academy Press. Washington DC. http//books.nap.edu/books/0309051266/html/index .html

Peters L.C. , Sist T.C. \& Kristal M.B. 1991. Maintenance and Decline of the Suppression of Infanticide in Mother Rats. Physiol \& Behav. 50 (2): $451-456$

Shively M.J. 1993. Anatomía Veterinaria Básica, Comparativa y Clínica. Editorial El Manual Moderno S.A, México D.F. : 341- 350.

Steuer M.A., Thompson A.C., Doerr J.C., Youakim M. \& Kristal M.B. 1987. Induction of Maternal Behavior in Rats: Effects of Pseudopregnancy Termination and Placenta-Smeared Pups. Behavioral Neuroscience. 101 (2): 219-227

Stylianopoulos C. 1999. Efectos de la fortificación y enriquecimiento de tortillas de maíz en el crecimiento y desarrollo cerebral de ratas durante dos generaciones. Tesis para optar el titulo de Master Scientiae. Instituto Tecnológico de Monterrey. Unidad de Postgrado. Maestría en Biotecnología. Monterrey - México. TRANSFERENCIA 48. Año 12. Número 48

http://www.mty.itesm.mx/die/ddre/transferencia/Trans ferencia48/eep-06.htm

Sumbera R., Burda H. \& Chitaukali W. 2003. Reproductive biology of a solitary subterranean Bathyergid Rodent, the Silvery Mole - Rat (Heliophobius argenteocinereus). Journal of Mammalogy. 84 (1): 278 - 287.

Vega M. 2000 . Género Rattus. Portal de Veterinaria. (ISSN 1667-507X)

http://www.portalveterinaria.com/sections.php?op=vie warticle\&artid $=7$

Veloso C. \& Bozinovic F. 2000. Effect of food quality on the energetics of reproduction in a precocial rodent, Octodon degus. Journal of Mammalogy. 81 (4): 971-978.

Zubritsky E. 1997. The Limits of Love. What is the biology of motherly love? And why does it sometimes break down? Endeavors Magazine.13 (2).

Zullinger E.M, Ricklefs R., Redford K.H. \& Mage G. M. 1984. Fitting sigmoidal equations to mammalian growth curves. Journal of Mammalogy. 65 (4): 607-636. 